CONFORMAL GEOMETRY AND DYNAMICS

An Electronic Journal of the American Mathematical Society

Volume 3, Pages 24-36 (March 11, 1999)

S $1088-4173(99) 00010-7$

\title{
DEFORMATION OF SCHOTTKY GROUPS IN COMPLEX HYPERBOLIC SPACE
}

\author{
BEAT AEBISCHER AND ROBERT MINER
}

\begin{abstract}
Let $G=P U(1, d)$ be the group of holomorphic isometries of complex hyperbolic space $\mathbf{H}_{\mathbf{C}}^{d}$. The latter is a Kähler manifold with constant negative holomorphic sectional curvature. We call a finitely generated discrete group $\Gamma=\left\langle g_{1}, \ldots, g_{n}\right\rangle \subset G$ a marked classical Schottky group of rank $n$ if there is a fundamental polyhedron for $G$ whose sides are equidistant hypersurfaces which are disjoint and not asymptotic, and for which $g_{1}, \ldots, g_{n}$ are side-pairing transformations. We consider smooth families of such groups $\Gamma_{t}=\left\langle g_{1, t}, \ldots, g_{n, t}\right\rangle$ with $g_{j, t}$ depending smoothly $\left(C^{1}\right)$ on $t$ whose fundamental polyhedra also vary smoothly. The groups $\Gamma_{t}$ are all algebraically isomorphic to the free group in $n$ generators, i.e. there are canonical isomorphisms $\phi_{t}: \Gamma_{0} \rightarrow \Gamma_{t}$. We shall construct a homeomorphism $\Psi_{t}$ of $\overline{\mathbf{H}}_{\mathbf{C}}^{d}=\mathbf{H}_{\mathbf{C}}^{d} \cup \partial \mathbf{H}_{\mathbf{C}}^{d}$ which is equivariant with respect to these groups:
\end{abstract}

$$
\phi_{t}(g) \circ \Psi_{t}=\Psi_{t} \circ g \quad \forall g \in \Gamma_{0}, \quad 0 \leq t \leq 1
$$

which is quasiconformal on $\partial \mathbf{H}_{\mathbf{C}}^{d}$ with respect to the Heisenberg metric, and which is symplectic in the interior. As a corollary, the limit sets of such Schottky groups of equal rank are quasiconformally equivalent to each other.

The main tool for the construction is a time-dependent Hamiltonian vector field used to define a diffeomorphism, mapping $D_{0}$ onto $D_{t}$, where $D_{t}$ is a fundamental domain of $\Gamma_{t}$. In two steps, this is extended equivariantly to $\overline{\mathbf{H}}_{\mathbf{C}}^{d}$.

The method yields similar results for real hyperbolic space, while the analog for the other rank-one symmetric spaces of noncompact type cannot hold.

\section{Introduction and Statement of Results}

Complex hyperbolic space $\mathbf{H}_{\mathbf{C}}^{d}$ is a complete Riemannian manifold with sectional curvature pinched between -2 and $-1 / 2$. A standard model for it is the unit ball $\mathbf{B} \subset \mathbf{C}^{d}(d \geq 2)$ with the Bergman metric. For the case $d=1$ note that $\mathbf{H}_{\mathbf{C}}^{1}$ is isomorphic to the real hyperbolic plane $\mathbf{H}_{\mathbf{R}}^{2}$. As a complex manifold, $\mathbf{H}_{\mathbf{C}}^{d}$ admits a natural Kähler structure, and therefore also a symplectic structure.

Received by the editors March 3, 1997 and, in revised form, November 4, 1998.

1991 Mathematics Subject Classification. Primary 30C65; Secondary 32G10, 57S30, 53C55, $58 \mathrm{~F} 05$.

Key words and phrases. Complex hyperbolic space, Schottky group, deformation, quasiconformal mapping, Heisenberg group.

B. Aebischer supported by Schweizerischer Nationalfonds.

R. Miner partially supported by NSF grant DMS-9404174.

(C)1999 American Mathematical Society 
The group of holomorphic isometries of $\mathbf{H}_{\mathbf{C}}^{d}$ is the Lie group $P U(1, d)$. G. D. Mostow [20] established that finite covolume discrete subgroups of $P U(1, d)$ are rigid. Recent work has shown that interesting phenomena still arise in connection with deformations of smaller discrete subgroups. (See for example [9], [10], [7].)

At the same time, a successful theory of quasiconformal mapping on complex hyperbolic space and its boundary has been developed by Korányi, Reimann, Pansu and others. (See for example [16] and [21].) Moreover, as in real hyperbolic geometry, there seems to be much fertile ground for the application of quasiconformal mappings to deformation questions about complex hyperbolic groups. Indeed, Mostow's rigidity theorem involves quasiconformal mapping in a vital way. In this article, we offer another application of quasiconformal mappings to deformation theory of groups that are far from having finite covolume. Specifically, we show that smooth deformations of proper complex hyperbolic Schottky groups are quasiconformally stable in an appropriate sense.

For comparison recall the definition of quasiconformal stability from the theory of Kleinian groups as given by Bers in [2]. Let $G$ be any subgroup of $P S L(2, \mathbf{C})$. A homomorphism $\chi: G \rightarrow P S L(2, \mathbf{C})$ will be called allowable if it preserves the square traces of parabolic and elliptic elements. Say that $\chi$ is a quasiconformal deformation of $G$ if there exists a quasiconformal mapping $w: \hat{\mathbf{C}} \rightarrow \hat{\mathbf{C}}$ such that $\chi(g)=w \circ g \circ w^{-1}$ for all $g \in G$. A finitely generated Kleinian group $\Gamma$ is then called quasiconformally stable if every allowable homomorphism sufficiently close to the identity is a quasiconformal deformation. In [2], Bers described a criterion (involving the quadratic differentials for $\Gamma$ ) to determine whether a group $\Gamma$ is quasiconformally stable. As a consequence of this criterion, it follows that Fuchsian groups, Schottky groups, groups of Schottky type and certain non-degenerate $B$ groups are all quasiconformally stable [3].

As with real hyperbolic isometries, elements of $P U(1, d)$ can be classified as loxodromic, parabolic or elliptic. Let $\Gamma=\left\langle g_{1}, \ldots, g_{n}\right\rangle \subset P U(1, d)$ be a discrete group generated by $n$ loxodromic elements. Assume $\Gamma$ admits a fundamental polyhedron $D$ whose sides are equidistant hypersurfaces (i.e. sets of the form $\mathcal{E}(x, y)=\{z \in \mathbf{B}: \rho(z, x)=\rho(z, y)\}$ for some $x$ and $y)$ such that $g_{1}, \ldots, g_{n}$ are side-pairing transformations for $D$. In analogy with Kleinian groups, if the sides of $D$ do not intersect and are not asymptotic, we call $\Gamma$ a classical Schottky group of rank $n$. When $\Gamma$ is given together with a choice of generators we refer to $\Gamma$ as a marked classical Schottky group.

Some authors define classical Schottky groups to include the case where the sides of $D$ are asymptotic. However, following Maskit [18] we term these groups of Schottky type, and focus our attention on groups where the sides of $D$ are disjoint on the interior of $\mathbf{B}$ and their closures are disjoint on the boundary.

The space of such groups (modulo conjugation) has real dimension $4+$ $d(n d+2 n-6)$. Namely, by [4, Lemma 3.2.2], a loxodromic element with given fixed points has $(d-1)^{2}+1$ real parameters left. Every fixed point contributes $2 d-1$ real parameters, and three fixed points can be normalized by one real parameter, namely Cartan's angular invariant (see e.g. $[8, \S 7]$ ). The limit set

$$
\Lambda=\overline{\Gamma x_{0}} \cap \partial \mathbf{B}
$$

of such a group is closed and totally disconnected (i.e. a Cantor set) (see e.g. [22, Theorem 12.1.21]). 
Consider a family $\left\{\Gamma_{t}: t \in[0,1]\right\}$ of marked classical Schottky groups with generators $g_{1, t}, \ldots, g_{n, t}$, depending $C^{1}$ on $t$, i.e. $g_{j, t}=P\left(\tilde{g}_{j, t}\right)$ with $t \mapsto \tilde{g}_{j, t} \in U(1, d)$ of class $C^{1}$, where $P: U(1, d) \longrightarrow P U(1, d)$ is the natural projection. Note that the $\Gamma_{t}$ are all isomorphic to the free group on $n$ generators (cf. [22, Theorem 12.1.19]), hence there are canonical isomorphisms

$$
\phi_{t}: \Gamma_{0} \stackrel{\simeq}{\longrightarrow} \Gamma_{t} .
$$

Given such a family $\left\{\Gamma_{t}\right\}$ of marked classical Schottky groups, suppose further that for each $t$ there exists a Schottky fundamental polyhedron $P_{t}$ for $\Gamma_{t}$ with sides $\left\{S_{1, t}, \ldots, S_{2 n, t}\right\}$ and with side-pairing transformations $g_{j, t}\left(S_{j, t}\right)=S_{j+n, t}$ where $j=1, \ldots, n$. When each family of equidistant hypersurfaces $\left\{S_{i, t}\right\}(i=1, \ldots, 2 n)$ is of class $C^{1}$ in $t$, we will say $\left\{\Gamma_{t}\right\}$ is a geometric deformation of $\Gamma=\Gamma_{0}$.

We show that geometric deformations of Schottky groups are quasiconformally stable in the following sense.

Theorem 1.1. Given such a geometric deformation $\left\{\Gamma_{t}\right\}$, for each $t$ there exists a homeomorphism $\Psi_{t}$ of $\overline{\mathbf{B}}$, which is equivariant:

$$
\phi_{t}(g) \circ \Psi_{t}=\Psi_{t} \circ g, \quad \forall g \in \Gamma_{0}, \quad t \in[0,1]
$$

and such that

(a) the restriction of $\Psi_{t}$ to $\mathbf{B}$ is a symplectic diffeomorphism and

(b) the restriction to $\partial \mathbf{B}$ is quasiconformal with respect to the Heisenberg metric.

Note that the boundary $\partial \mathbf{B}$ of complex hyperbolic space can be identified with the one-point compactification of the Heisenberg group $H^{d-1}$, see e.g. [8, §2.1], [14] or the next section. The Heisenberg metric on $H^{d-1} \simeq \mathbf{C}^{d-1} \times \mathbf{R}$ is also defined in Section 2.

For the purpose of finding quasiconformal equivalences between groups, the condition that a deformation be geometric is perhaps not as restrictive as it first might seem. For example, if $\left\{\Gamma_{t}\right\}$ is any $C^{1}$ deformation of classical Schottky groups, there is some interval $[0, \epsilon]$ on which $\left\{\Gamma_{t}\right\}$ is geometric, since

$$
\left\{S_{1,0}, \ldots, S_{n, 0}, g_{1, t}\left(S_{1,0}\right), \ldots, g_{n, t}\left(S_{n, 0}\right)\right\}
$$

is a smooth family of Schottky fundamental polyhedra on some interval.

Geometric deformations are also sufficiently prevalent to connect any two marked classical Schottky groups of the same rank (provided $d>1$ ) since we may define a deformation of groups by constructing a deformation of fundamental polyhedra. Thus, we have the following corollary.

Corollary 1.2. Given two marked classical Schottky groups $\Gamma_{0}$ and $\Gamma_{1}$ of equal rank there exists a homeomorphism $\Psi$ of $\overline{\mathbf{B}}$ such that $\Gamma_{0}=\Psi^{-1} \Gamma_{1} \Psi$ and such that its restriction to the boundary $\partial \mathbf{B}$ is quasiconformal and the restriction to $\mathbf{B}$ is a symplectic diffeomorphism.

In particular, the limit sets $\Lambda_{0}$ and $\Lambda_{1}$ are quasiconformally equivalent: $\Lambda_{1}=$ $\Psi\left(\Lambda_{0}\right)$. Because the image of a Schottky group under a homomorphism close to the identity is again a Schottky group of the same rank, the quasiconformal stability expressed by Theorem 1.1 includes quasiconformal stability in the sense of Bers.

In real hyperbolic space $\mathbf{H}_{\mathbf{R}}^{d}$, the following is known. For $d=3$, members of a continuously parametrized family of finitely generated discrete groups are canonically isomorphic to each other, [13, Theorem 3]. In the other direction, and in arbitrary dimension, Tukia [23] has shown that a type preserving isomorphism between 
two geometrically finite Möbius groups is induced by a canonical quasisymmetric mapping on their limit sets. Again for $d=3$, using the generalized $\lambda$-lemma of holomorphic motions, one can show: If $\left(\Gamma_{z}\right)_{z \in \Delta}$ is a holomorphic family of finitely generated Kleinian groups such that no new parabolic elements are created as $z$ varies in the unit disk, then the $\Gamma_{z}$ are canonically quasiconformally conjugate on their limit sets, [1]. In fact, the equivariant version of the generalized $\lambda$-lemma by Earle, Kra and Krushkal' [6] shows that the groups $\Gamma_{z}$ are quasiconformally conjugate on $\partial \mathbf{H}_{\mathbf{R}}^{3} \simeq \hat{\mathbf{C}}$.

The method used in the present paper yields analogous results for classical Schottky groups in $\mathbf{H}_{\mathbf{R}}^{d}$ for $d \geq 3$. Use the ball $\mathbf{B} \subset \mathbf{R}^{d}$ as a model for real hyperbolic space and on its boundary $\partial \mathbf{B}$ use the euclidean (chordal) metric to define quasiconformality. In the interior, it does not matter whether one uses the hyperbolic or the euclidean metric to define quasiconformality, because these metrics are conformally equivalent.

Theorem 1.3. Given a geometric deformation $\left\{\Gamma_{t}\right\}, 0 \leq t \leq 1$, of classical Schottky groups acting on $\mathbf{B} \subset \mathbf{R}^{d}$ there exist homeomorphisms $\Psi_{t}$ of $\overline{\mathbf{B}}$ such that (1.1) holds, $\Psi_{t}$ is a quasiconformal diffeomorphism of $\mathbf{B}$, and the restriction to $\partial \mathbf{B}$ is quasiconformal as well.

As a corollary, marked classical Schottky groups of equal rank $n$ (acting on $\mathbf{H}_{\mathbf{R}}^{d}$, $d \geq 3$ ) are quasiconformally conjugate. The hyperbolic quotient manifolds $\mathbf{B} / \Gamma_{0}$ and $\mathbf{B} / \Gamma_{1}$ are quasiconformally equivalent. By [18, VIII.D.1], for $d=3$ these manifolds are 'handlebodies of genus $n$ '.

By the known rigidity of quaternionic hyperbolic space and the Cayley plane, analogous results for Schottky groups on these spaces cannot hold. Namely, as P. Pansu has shown, every quasiconformal map on the boundary of quaternionic hyperbolic space or the Cayley plane is the extension of an isometry (Corollary 11.2 and Proposition 11.5 in [21]). Thus, for the result to hold, the space of marked classical Schottky groups of rank $n$ modulo conjugation by isometries would have to reduce to a point, which of course is not the case. However, in the quaternionic and Cayley cases our construction still yields an equivariant homeomorphism of hyperbolic space and its boundary which is a diffeomorphism in the interior.

In the general case of negatively curved groups, Martin has shown [17, Thm. 5.2] that a continuous deformation of a torsion free group through discrete cocompact groups is topologically trivial.

Section 2 contains introductory material and some lemmas which are used in Section 3 to prove the theorems. It is a pleasure for us to thank Martin Reimann for many helpful discussions.

\section{Notation And Preliminaries}

In this section, $\mathbf{B}$ shall always be the unit ball in $\mathbf{C}^{d}$. As noted above, $\mathbf{B}$ is a model of $\mathbf{H}_{\mathbf{C}}^{d}$ when supplied with the Bergman metric $g$ :

$$
g=\frac{2}{d+1} \operatorname{Re} \sum_{j, k=1}^{d} \frac{\partial^{2} \log K(z)}{\partial z_{j} \partial \bar{z}_{k}} d z_{j} \otimes d \bar{z}_{k}
$$

where

$$
K(z)=K_{\mathbf{B}}(z, z)=\frac{d !}{\pi^{d}}\left(1-|z|^{2}\right)^{-(d+1)}
$$


is the Bergman kernel function. The distance function defined by $g$, i.e. the hyperbolic metric, is denoted by $\rho$. The group of holomorphic isometries of $\mathbf{H}_{\mathbf{C}}^{d}$ is

$$
G=P U(1, d) .
$$

Here, $U(1, d)$ is the subgroup of $G L(d+1, \mathbf{C})$ leaving invariant the hermitian form

$$
\langle y, y\rangle=-y_{0} \bar{y}_{0}+y_{1} \bar{y}_{1}+\cdots+y_{d} \bar{y}_{d}, \quad y=\left(y_{0}, y_{1}, \ldots, y_{d}\right) \in \mathbf{C}^{d+1} .
$$

It acts linearly on $\mathbf{C}_{-}^{d+1}=\left\{y \in \mathbf{C}^{d+1}:\langle y, y\rangle<0\right\}$. By definition, $\mathbf{H}_{\mathbf{C}}^{d}$ is the space of complex lines in $\mathbf{C}_{-}^{d+1}$, which in inhomogeneous coordinates

$$
w_{j}=\frac{y_{j}}{y_{0}} \quad(j=1, \ldots, d)
$$

corresponds to the ball B. Dividing by the center $Z=\{\lambda I:|\lambda|=1\}$ of $U(1, d)$, we get the quotient $P U(1, d)=U(1, d) / Z$, which acts effectively on $\mathbf{B}$.

Alternatively, $\mathbf{H}_{\mathbf{C}}^{d}$ is the rank one symmetric space $G / K$, where $K=$ $P(U(1) \times U(d)) \simeq U(d)$ is the stabilizer of $0 \in \mathbf{B}$. The symmetry at 0 is just $z \mapsto-z$. The metric $g$ can be defined at $z=0$ by the Killing form of the Lie algebra of $G$ and then be transported everywhere by elements of $G$.

Together with the natural complex structure $J$, complex hyperbolic space also carries a symplectic structure

$$
\omega(X, Y)=g(J X, Y),
$$

i.e. $d \omega=0$, and $\mathbf{H}_{\mathbf{C}}^{d}$ is a Kähler manifold.

The Heisenberg group arises as the 'translation part' of the stabilizer of the point $p=(0, \ldots, 0,-1)$ of the action of $G$ on $\partial \mathbf{B}$. The Iwasawa decomposition of this stabilizer is $M A N$, where $M \simeq U(d-1), A \simeq \mathbf{R}_{+}$, and $N$ is the Heisenberg group, see [14]. The group $N$ acts simply transitively on $\partial \mathbf{B} \backslash\{p\}$, hence its onepoint compactification $\hat{N}$ can be identified with $\partial \mathbf{B}$. In suitable coordinates, the Heisenberg group $N$ is

$$
H^{d-1}=\mathbf{C}^{d-1} \times \mathbf{R}
$$

with the group law

$$
(z, t) \cdot\left(z^{\prime}, t^{\prime}\right)=\left(z+z^{\prime}, t+t^{\prime}+2 \operatorname{Im}\left(z \bar{z}^{\prime}\right)\right) .
$$

The Heisenberg metric $d$ is defined by

$$
d(g, h)=\left|g^{-1} h\right|
$$

where

$$
|g|=\left(|z|^{4}+t^{2}\right)^{1 / 4}, \quad g=(z, t) \in H^{d-1} .
$$

By definition it is left-invariant. Via stereographic projection, $d$ is conformally equivalent to the metric $d_{S}$ on $\partial \mathbf{B}$,

$$
d_{S}(z, w)=|1-(z, w)|^{1 / 2}, \quad(z, w)=\sum_{j=1}^{d} z_{j} \bar{w}_{j},
$$

see $[14$, section F]. Thus, we say a homeomorphism of $\partial \mathbf{B}$ is 'quasiconformal with respect to the Heisenberg metric' if and only if it is quasiconformal with respect to $d_{S}$. 
For two points $x, y \in \mathbf{B}$, let

$$
\mathcal{E}(x, y)=\{z \in \mathbf{B}: \rho(z, x)=\rho(z, y)\}
$$

be the equidistant hypersurface separating them. A $C^{1}$-family of equidistant hypersurfaces $\left\{S_{t}\right\}_{t \in[0,1]}$ is given by $S_{t}=\mathcal{E}\left(x_{t}, y_{t}\right)$ with $x_{t}, y_{t} \in \mathbf{B}$ of class $C^{1}$ in $t$. In order to deform fundamental domains for Schottky groups, we will need the lemma below relating $C^{1}$-families of equidistant hypersurfaces to flows. Recall that a continuously time dependent smooth vector field $X_{t}$ defined for $0 \leq t \leq 1$ on a manifold $M$ generates a two-parameter family of diffeomorphisms $\psi_{s, t}, 0 \leq s, t \leq 1$ such that

$$
\psi_{s, t} \circ \psi_{r, s}=\psi_{r, t} \quad \text { and } \quad \psi_{t, t}=i d_{M},
$$

provided $M$ is compact or $X$ does not grow too fast at infinity. To be specific, $\psi_{s, t}(x)$ is defined as $y(t)$, where $y$ is the solution of $\frac{d y}{d \tau}=X_{\tau}(y(\tau))$ with $y(s)=x$. For simplicity we shall call the family $\psi_{s, t}$ the flow generated by $X_{t}$, even though it is not a flow in the usual sense.

Lemma 2.1. Let $S_{t}, t \in[0,1]$, be a $C^{1}$-family of equidistant hypersurfaces. Then there exists a continuously time dependent Hamiltonian vector field $X_{t}$ on $\mathbf{B}$ generating a flow $\psi_{s, t}$ of isometries such that $\psi_{0, t}\left(S_{0}\right)=S_{t}$. (Equivalently, $\psi_{s, t}\left(S_{s}\right)=$ $S_{t}$.) The Hamiltonian is continuous in $t$ and $C^{\infty}$ in $x$, and $X_{t}$ extends smoothly to $\overline{\mathbf{B}}$.

Proof. Let $\gamma_{t}$ be the (real) geodesic through $x_{t}$ and $y_{t}$. There exist unique points $u_{t}, v_{t} \in \gamma_{t}$ such that

$$
\mathcal{E}\left(u_{t}, v_{t}\right)=S_{t}, \quad \rho\left(u_{t}, v_{t}\right)=\rho\left(x_{0}, y_{0}\right), \quad \rho\left(u_{t}, x_{t}\right)<\rho\left(u_{t}, y_{t}\right) .
$$

Because $\rho\left(x_{t}, y_{t}\right)$ is bounded from below, the midpoint of the geodesic arc $\left[x_{t}, y_{t}\right]$ and the endpoints of $\gamma_{t}$ are $C^{1}$ in $t$. Hence $u_{t}$ and $v_{t}$ are $C^{1}$ in $t$.

Assume $h_{t} \in G$ satisfies

$$
h_{t}\left(x_{0}\right)=u_{t}, \quad h_{t}\left(y_{0}\right)=v_{t} .
$$

Then

$$
h_{t}\left(S_{0}\right)=h_{t}\left(\mathcal{E}\left(x_{0}, y_{0}\right)\right)=\mathcal{E}\left(u_{t}, v_{t}\right)=S_{t} .
$$

Let $H \subset G$ be the subgroup fixing $x_{0}$ and $y_{0}$ and let $\pi: G \rightarrow G / H$ be the canonical projection. (By [4, Lemma 3.2.2], $H$ is a closed compact subgroup isomorphic to $P(U(1) \times U(d-1)) \simeq U(d-1)$.) The projection $\pi\left(h_{t}\right)$ is uniquely determined by the condition (2.1). Hence with any choice of $\left\{h_{t}\right\}_{t \in[0,1]}$ satisfying $(2.1), \pi\left(h_{t}\right)$ is $C^{1}$ in $t$, because $u_{t}$ and $v_{t}$ are $C^{1}$. Since $H$ is a submanifold of $G$, it is easy to see in local coordinates that we can choose a $C^{1}$-lift of the curve $\pi\left(h_{t}\right)$ which is the identity at $t=0$. We denote this lift again by $h_{t}$.

Now we define the vector field $X_{t}$ on $\overline{\mathbf{B}}$ by

$$
X_{t}\left(h_{t}(x)\right)=\left.\frac{d}{d s}\right|_{s=t} h_{s}(x) .
$$

Because $h_{t}$ is an isometry and is biholomorphic, it is a symplectomorphism of $\mathbf{B}$. Thus, the inner product $\left.X_{t}\right\lrcorner \omega$ is closed:

$$
\left.d\left(X_{t}\right\lrcorner \omega\right)=\left.\frac{d}{d s}\right|_{s=t}\left(h_{s} \circ h_{t}^{-1}\right)^{*} \omega=0 .
$$


(See for instance [11], formula (22.1).) In other words, the vector field $X_{t}$ is locally Hamiltonian. But $\mathbf{B}$ is simply connected, so there exists a global Hamiltonian for $X_{t}$. From the definition of the vector field we see that it is continuous in time. In the variable $x \in \overline{\mathbf{B}}$ it is $C^{\infty}$, because the action $\Phi: G \times B \rightarrow B, \Phi(g, x)=g x$ is smooth in both variables:

$$
X_{t}(x)=\left.\frac{d}{d s}\right|_{s=t} h_{s}\left(h_{t}^{-1}(x)\right)=\left.\frac{d}{d s}\right|_{s=t} \Phi\left(h_{s} h_{t}^{-1}, x\right)=D_{1} \Phi(e, x) Y_{t},
$$

where $Y_{t}=\left.\frac{d}{d s}\right|_{s=t} h_{s} h_{t}^{-1}$ is an element of the Lie algebra of $G$, only depending on $t$. Since the Hamiltonian is locally determined up to a constant by $\left.X_{t}\right\lrcorner \omega=d H_{t}$, it can be chosen continuous in $t$ and $C^{\infty}$ in $x$.

We shall also need a lemma about extending Hamiltonian vector fields. Again we begin with some notation. A defining function (or Kähler potential) for $\mathbf{B}$ is $\rho(z)=1-|z|^{2}$, that is, $\mathbf{B}=\left\{z \in \mathbf{C}^{d}: \rho(z)>0\right\}$. The contact structure on $\partial \mathbf{B}$ is given by the contact form $\theta=-J d \rho$ and the Reeb vector field $T$ is defined by $T\lrcorner d \theta=0$ and $\theta(T)=1$. The Levi form on the horizontal bundle $\operatorname{ker} \theta \subset T \partial \mathbf{B}$ is given by

$$
L(X, Y)=-d \theta(X, J Y)
$$

and it is positive definite, i.e. $\mathbf{B}$ is a strictly pseudoconvex domain. Finally, the horizontal gradient $\nabla_{0}$ is defined by

$$
L\left(\nabla_{0} p, X\right)=X p
$$

for a differentiable function $p$ on $\mathbf{B}$ and $X \in \operatorname{ker} \theta$.

Lemma 2.2. Suppose $\sigma: \overline{\mathbf{B}} \rightarrow[0,1]$ is smooth and $H$ is a smooth Hamiltonian on $\mathbf{B}$ such that the vector field it generates extends smoothly to a (tangential) vector field on $\partial \mathbf{B}$. Then the vector field $X$ generated by the Hamiltonian $\sigma H$ extends smoothly to $\partial \mathbf{B}$, too, and is given there by

$$
X=-J \nabla_{0} p+p T
$$

where the contact Hamiltonian (or the potential)

$$
p(z)=\sigma(z) \lim _{x \rightarrow z} \rho(x) H(x)
$$

is a smooth real function on $\partial \mathbf{B}$.

Proof. By [15, Proposition 1], the flow of $H$ extends to a contact deformation on $\partial \mathbf{B}$, i.e. the extension of the vector field $-J \nabla H$ to $\partial \mathbf{B}$ equals $-J \nabla_{0} \tilde{p}+\tilde{p} T$ for some smooth function $\tilde{p}: \partial \mathbf{B} \rightarrow \mathbf{R}$. The following calculation is the same as in the proof of Proposition 3 in [15]. In a neighborhood of $\partial \mathbf{B}$ set $Z_{1}=(T-i J T) / 2$ and supplement $Z_{1}$ to an orthogonal basis $\left\{Z_{1}, \ldots, Z_{d}\right\}$ of $T^{1,0} \mathbf{B}$ (with respect to $g$ extended as a Hermitian metric on $\left.T \mathbf{B}^{\mathbf{C}}\right)$ normalized such that $L\left(Z_{j}, Z_{k}\right)=\delta_{j, k}$ for $j, k \in\{2, \ldots, d\}$. Then calculation yields

$$
\begin{aligned}
-J \nabla H & =i \sum_{j=2}^{d}\left[\left(Z_{j}(\rho H)\right) \bar{Z}_{j}-\left(\bar{Z}_{j}(\rho H)\right) Z_{j}\right]+ \\
& \frac{i}{\rho\left\|Z_{1}\right\|^{2}}\left[\left(Z_{1}(\rho H)\right) \bar{Z}_{1}-\left(\bar{Z}_{1}(\rho H)\right) Z_{1}\right]+\frac{\rho H}{2 \rho^{2}\left\|Z_{1}\right\|^{2}} T .
\end{aligned}
$$

Note that we got rid of the factor $2 /(d+1)$ in [15], because of our different normalization of the symplectic structure: $\omega=d J d \log \rho$ instead of $\omega=-1 / 2 d J d \log \rho^{-(d+1)}=$ 
$(d+1) / 2 d J d \log \rho$. (With the normalization used here, the holomorphic sectional curvature is -2 , cf. [8].)

Now, $2 \rho^{2}\left\|Z_{1}\right\|^{2}=1+O(\rho)$ as one approaches the boundary (cf. [15]). It follows that $\lim _{x \rightarrow z} \rho(x) H(x)=\tilde{p}(z)$. Thus, $p(z)=\sigma(z) \lim _{x \rightarrow z} \rho(x) H(x)$ is $C^{\infty}$ on $\partial \mathbf{B}$ and the formula (2.2) with $H$ replaced by $\sigma H$ shows the extension of $X=-J \nabla(\sigma H)$ to $\partial \mathbf{B}$ is given by $-J \nabla_{0} p+p T$.

\section{Proof of the Theorems}

Proof of Theorem 1.1. We are given a geometric deformation of classical Schottky groups $\Gamma_{t}=\left\langle g_{1, t}, \ldots, g_{n, t}\right\rangle$. Set $g_{-j, t}=g_{j, t}^{-1}$. Let $D_{t}$ denote the associated Schottky fundamental polyhedra for $\Gamma_{t}$, where $g_{j, t}\left(S_{-j, t}\right)=S_{j, t}$ for $j=1, \ldots, n$. Since $\left\{D_{t}\right\}$ is by definition a $C^{1}$ family of Schottky polyhedra, the sides of $D_{t}$ are given by

$$
S_{j, t}=\mathcal{E}\left(x_{j, t}, y_{j, t}\right), \quad j= \pm 1, \ldots, \pm n
$$

where $x_{j, t}$ and $y_{j, t}$ are $C^{1}$ in $t$.

By Lemma 2.1 we have time dependent Hamiltonians $H_{j}$ generating isometries $h_{j, t}$ mapping $S_{j, 0}$ onto $S_{j, t}$ for $j=1, \ldots, n$. If we set

$$
h_{-j, t}=g_{j, t}^{-1} \circ h_{j, t} \circ g_{j, 0},
$$

then $h_{-j, t}$ is an isometry mapping $S_{-j, 0}$ onto $S_{-j, t}$ for $j=1, \ldots, n$. Since $h_{-j, t}$ is symplectic, there exists a global time dependent Hamiltonian $H_{-j}$ for it as in the proof of Lemma 2.1.

For $j= \pm 1, \ldots, \pm n$ choose cutoff functions $\sigma_{j}:[0,1] \times \overline{\mathbf{B}} \rightarrow[0,1]$ which are $C^{1}$ in the first argument and $C^{\infty}$ in the second. Moreover, require that $\sigma_{j, t}=\left.\sigma_{j}\right|_{\{t\} \times \mathbf{B}}$ vanishes outside some euclidean neighborhood of $S_{j, t}$, that it is identically one in a smaller neighborhood, and that the supports of the $\sigma_{j}$ are disjoint. (Remember the sides of $D_{t}$ do not intersect.) We can also assume that

$$
\sigma_{j, t}=\sigma_{j, 0} \circ h_{j, t}^{-1} \quad \text { and } \quad \sigma_{-j, t}=\sigma_{j, t} \circ g_{j, t}
$$

for $j= \pm 1, \ldots, \pm n$. In fact, it suffices to choose smooth functions $\sigma_{j, 0}(j=1, \ldots, n)$ with sufficiently small supports and then define $\sigma_{j, t}$ by these conditions. The diffeomorphism $\psi_{t} \equiv \psi_{0, t}$ generated by the time dependent Hamiltonian

$$
H=\sum_{j=1}^{n}\left(\sigma_{j} H_{j}+\sigma_{-j} H_{-j}\right)
$$

then maps each side of $D_{0}$ onto the corresponding side of $D_{t}$. Applying Lemma 2.2 to every summand in the above equation (frozen to a fixed time $t$ ), we see that $-J \nabla H$ and hence $\psi_{t}$ extends smoothly to the boundary $\partial \mathbf{B}$.

Let

$$
F_{t}=D_{t} \cup \bigcup_{j=1}^{n} S_{j, t}
$$

be a fundamental set for the action of $\Gamma_{t}$ on $\mathbf{B}$. Adding the points on the boundary at infinity adhering to $F_{t}$, we get a fundamental set $\bar{F}_{t}$ for the action of $\Gamma_{t}$ on $\mathbf{B} \backslash \Lambda_{t}$, where $\Lambda_{t}$ is the limit set of $\Gamma_{t}$. It follows that $\psi_{t}\left(\bar{F}_{0}\right)=\bar{F}_{t}$.

We can now extend $\left.\psi_{t}\right|_{\bar{F}_{0}}$ to an equivariant map $\Psi_{t}$ on $\overline{\mathbf{B}} \backslash \Lambda_{0}$ as follows. For $x \in \overline{\mathbf{B}} \backslash \Lambda_{0}$ there is a unique element $h$ of $\Gamma_{0}$ such that $h x \in \bar{F}_{0}$, we set

$$
\Psi_{t}(x)=\phi_{t}(h)^{-1} \psi_{t}(h x) .
$$


Away from the boundary of translates of the fundamental set, $\Psi_{t}$ is clearly a diffeomorphism. Further, on the neighborhood $\left\{x \in \overline{\mathbf{B}}: \sigma_{j, 0}(x)=1\right\}$ of $S_{j, 0}$ one has

$$
g_{j, t} \circ \psi_{t} \circ g_{j, 0}^{-1}=g_{j, t} \circ h_{-j, t} \circ g_{j, 0}^{-1}=h_{j, t}=\psi_{t} .
$$

Thus, the extension $\Psi_{t}$ is indeed a diffeomorphism $\overline{\mathbf{B}} \backslash \Lambda_{0} \rightarrow \overline{\mathbf{B}} \backslash \Lambda_{t}$. Moreover, $\Psi_{t}$ agrees with $\psi_{t}$ on a neighborhood of $\bar{F}_{0}$, and hence

$$
\Psi_{t}\left(\bar{F}_{0}\right)=\bar{F}_{t}
$$

holds. One also easily verifies that the equivariance condition (1.1) is satisfied.

We now show $\Psi_{t}$ extends to a homeomorphism of $\overline{\mathbf{B}}$. For Schottky groups a point in the limit set corresponds to a unique infinite word in the generators (written from left to right). That is, to the point $y \in \Lambda_{0}$ corresponds the sequence $\left(g_{j}\right)$ such that $y=\lim g_{j}(0)$ and $g_{j}$ is a word of length $j$ in the generators of $\Gamma_{0}$. If we set

$$
\Psi_{t}(y)=\lim _{j \rightarrow \infty} \phi_{t}\left(g_{j}\right)(0) \in \Lambda_{t},
$$

it is clear that $\left.\Psi_{t}\right|_{\Lambda_{0}}$ is one-to-one and continuous, because nearby limit points correspond to words that have long equal beginnings. An arbitrary sequence in $\overline{\mathbf{B}} \backslash \Lambda_{0}$ converging to $y \in \Lambda_{0}$ can be written (uniquely) as $\left(g_{j}\left(x_{j}\right)\right)$, with $x_{j} \in \bar{F}_{0}$ and $g_{j} \in \Gamma_{0}$. Then the line

$$
\lim \Psi_{t}\left(g_{j}\left(x_{j}\right)\right)=\lim \phi_{t}\left(g_{j}\right) \Psi_{t}\left(x_{j}\right)=\lim \phi_{t}\left(g_{j}\right)(0)=\Psi_{t}(y)
$$

shows that the extension $\Psi_{t}$ is continuous on $\overline{\mathbf{B}}$. Changing the roles of the times 0 and $t$ yields the same for $\Psi_{t}^{-1}$. Thus $\Psi_{t}$ is a homeomorphism.

Finally, it remains to show that $\Psi_{t}$ is quasiconformal with respect to the Heisenberg metric on $\partial \mathbf{B}$. We begin by showing that $\Psi_{t}$ is quasiconformal on $\partial \mathbf{B} \backslash \Lambda_{0}$. By Lemma $2.2,\left.\psi_{t}\right|_{\partial \mathbf{B}}$ is the $[0,1]$-flow of the continuously time dependent vector field $-J \nabla_{0} p+p T$, where $p:[0,1] \times \partial \mathbf{B} \rightarrow \mathbf{R}$ is $C^{\infty}$ in its second argument and is given by

$$
\begin{aligned}
p & =\sum_{j=1}^{n}\left(\sigma_{j} p_{j}+\sigma_{-j} p_{-j}\right), \\
p_{j}(t, z) & =\lim _{x \rightarrow z} \rho(x) H_{j}(t, x), \quad j= \pm 1, \ldots, \pm n .
\end{aligned}
$$

Proposition 25 in [16] (which holds as well for time dependent potentials $p$ ) now implies $\left.\psi_{t}\right|_{\partial \mathbf{B}}$ is $K$-quasiconformal with $K$ depending only on the first and second horizontal derivatives of the cutoff functions $\sigma_{j}$ and the first horizontal derivatives of $p_{j}$. (Note that the second horizontal derivatives of $p_{j}$ vanish, because the flow generated by $p_{j}$ is conformal.) By the definition (3.1) of the extension $\Psi_{t}$ it immediately follows that $\Psi_{t}$ is quasiconformal on $\partial \mathbf{B} \backslash \Lambda_{0}$, since elements of $P U(1, d)$ operate conformally on $\partial \mathbf{B}$.

To show that quasiconformality extends across $\Lambda_{0}$, we use the analytic definition [16]. Since $\Lambda_{0}$ has Lebesgue measure zero, we only have to show the extension of $\Psi_{t}$ to $\partial \mathbf{B}$ has the ACL property, i.e. for every smooth fibration $\mathcal{F}$ by horizontal curves, $\Psi_{t}$ is absolutely continuous on almost every curve in $\mathcal{F}$. 
Consider first the case where the limit set $\Lambda_{0}$ has zero $(2 d-1)$-dimensional Hausdorff measure. Then the set of curves in $\mathcal{F}$ meeting $\Lambda_{0}$ has measure zero. To see this, note that by definition, the measure on $\mathcal{F}$ has the property

$$
c_{0} r^{2 d-1}<\text { measure }(\{\gamma \in \mathcal{F}: \gamma \cap B(x, r) \neq \emptyset\})<c_{1} r^{2 d-1},
$$

see [16]. Hence $\left.\Psi_{t}\right|_{\partial \mathbf{B}}$ inherits the ACL property from $\left.\Psi_{t}\right|_{\partial \mathbf{B} \backslash \Lambda_{0}}$.

In the general case, we extend the family $\Gamma_{t}$ up to $t=2$ in such a way that the generators of $\Gamma_{2}$ all preserve $S^{1}=\partial \mathbf{B} \cap(\mathbf{C} \times\{0\})$. The limit set $\Lambda_{2}$ then is contained in $S^{1}$ and has Hausdorff dimension at most two (the direction transverse to the contact hyperplane counts twice, see [5, p. 526] or [19]). In particular, $\Lambda_{2}$ has zero $(2 d-1)$-dimensional Hausdorff measure. Now set $\tilde{\Gamma}_{t}=\Gamma_{2-t}(0 \leq t \leq 2)$. As in the special case above, we can construct homeomorphisms $\tilde{\Psi}_{t}: \overline{\mathbf{B}} \rightarrow \overline{\mathbf{B}}$ with the required regularity properties and such that

$$
\tilde{\phi}_{t}(g) \circ \tilde{\Psi}_{t}=\tilde{\Psi}_{t} \circ g \quad \forall g \in \tilde{\Gamma}_{0}=\Gamma_{2},
$$

where $\tilde{\phi}_{t}=\phi_{2-t} \circ \phi_{2}^{-1}: \tilde{\Gamma}_{0} \stackrel{\simeq}{\longrightarrow} \tilde{\Gamma}_{t}$. One immediately verifies that $\Psi_{t}=\tilde{\Psi}_{2-t} \circ \tilde{\Psi}_{2}^{-1}$ satisfies (1.1) as well as the regularity properties claimed in Theorem 1.1.

Proof of Theorem 1.3. The proof goes along the same lines as the previous one, except that one gets quasiconformality much easier. So we just sketch the differences.

The analog of Lemma 2.1 holds, where the vector field $X_{t}$ is not Hamiltonian, but is defined on the closure $\overline{\mathbf{B}}$. Instead of cutting off Hamiltonians we cut off the time dependent vector fields $X_{j}$, which generate isometries mapping $S_{j, 0}$ onto $S_{j, t}$. Then the diffeomorphism $\psi_{t} \equiv \psi_{0, t}$ generated by the vector field $X=\sum_{j=1}^{n}\left(\sigma_{j} X_{j}+\sigma_{-j} X_{-j}\right)$ maps each side of the Schottky fundamental domain $D_{0}$ onto the corresponding side of $D_{t}$. The restriction $\left.\psi_{t}\right|_{\bar{F}_{0}}$ is extended as before to an equivariant diffeomorphism $\Psi_{t}: \overline{\mathbf{B}} \backslash \Lambda_{0} \rightarrow \overline{\mathbf{B}} \backslash \Lambda_{t}$. As before, $\Psi_{t}$ extends as a homeomorphism to $\partial \mathbf{B}$.

Now, $\psi_{t}$ is a diffeomorphism, hence is quasiconformal on $\mathbf{B}$ and on $\partial \mathbf{B}$. Hence the extension $\Psi_{t}$ of $\left.\psi_{t}\right|_{\bar{F}_{0}}$ is quasiconformal on $\mathbf{B}$ and on $\partial \mathbf{B} \backslash \Lambda_{0}$. As at the end of the previous proof, we only need to consider the case when $\Lambda_{0}$ is 'small', for instance when it has $\sigma$-finite $(d-2)$-dimensional Hausdorff measure. Then Theorem 35.1 in [24] shows that $\Psi_{t}$ is quasiconformal on all of $\partial \mathbf{B}$. Alternatively, one can directly apply Theorem 4.2 of [12] without having to make $\Lambda_{0}$ small.

\section{IlLustrations}

The following illustrations depict the boundaries of two Schottky fundamental polyhedra in $\mathbf{H}_{\mathbf{C}}^{2}$. Both pictures show fundamental polyhedra for Schottky groups of rank 2. The colored, deformed "spheres" are the boundaries of equidistant bisectors (sometimes called spinal spheres) which form the faces of the fundamental polyhedra.

The illustrations were generated using Mathematica 3.0 and Geomview on a Silicon Graphics work station. 


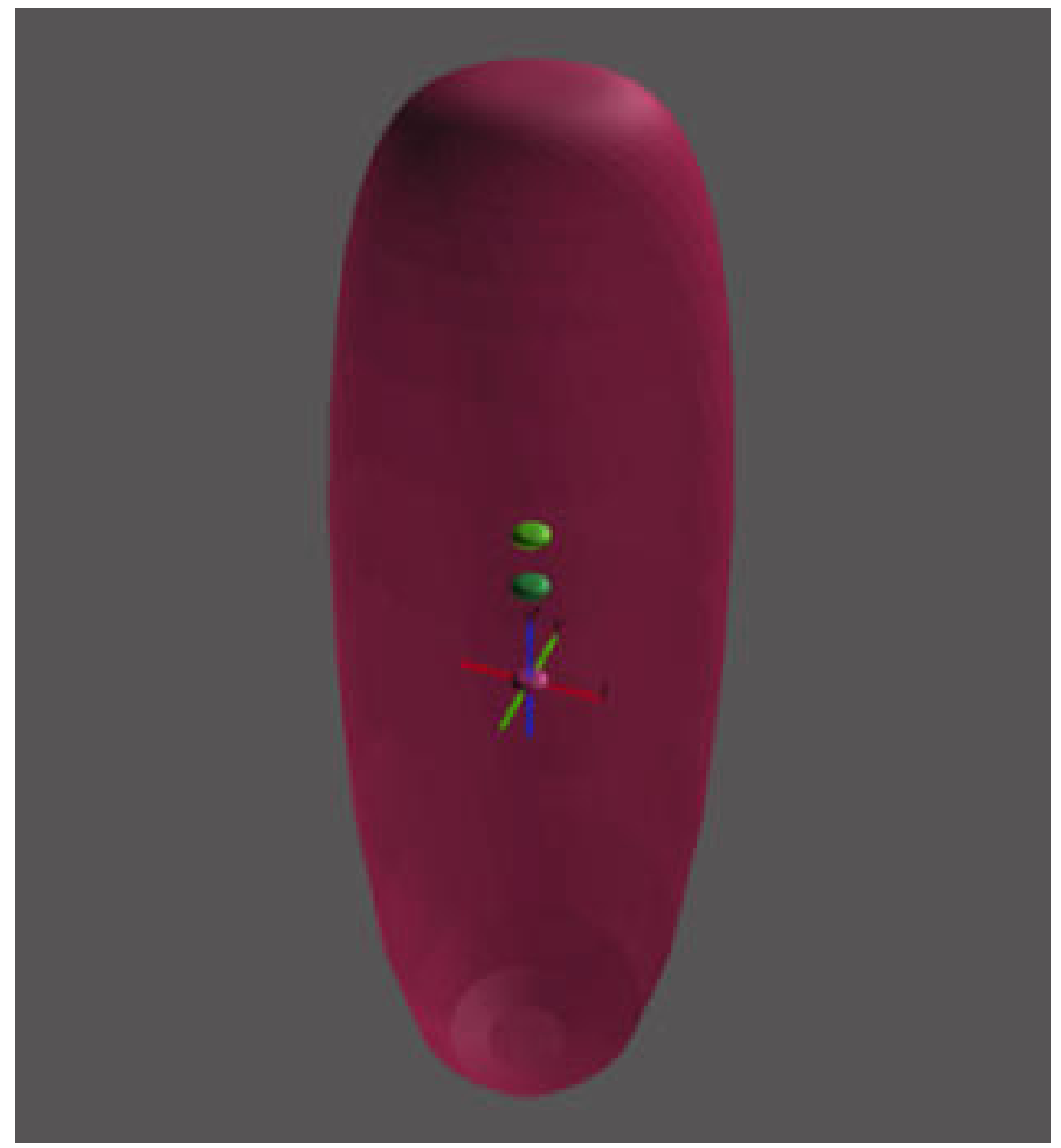

In this illustration, the fixed points of the first loxodromic generators lie at $(0,0)$ and infinity in Heisenberg coordinates. In Heisenberg coordinates, this generator acts as the dilation $(z, t) \mapsto(10 z, 100 t)$, and is the side pairing for the reddish spinal spheres. The second generator has fixed points at $(0,3)$ and $(0,4)$ in Heisenberg coordinates, and is the side paring for the greenish spinal spheres. It is conjugate to the first generator in $P U(2,1)$.

Note that intersection with the boundary of the Schottky fundamental polyhedron lies inside the large red spinal sphere, and outside the others. The group illustrated here is reasonably generic in the sense that its generators have fairly large dilation factors, and hence the sides of the fundamental polyhedron are far apart. On the other hand, in another sense, this group is quite special, since the fixed points of its generators all lie on a single chain (the vertical axis here) which results in an unusually regular, symmetric picture. In particular, all the spinal spheres are convex. 


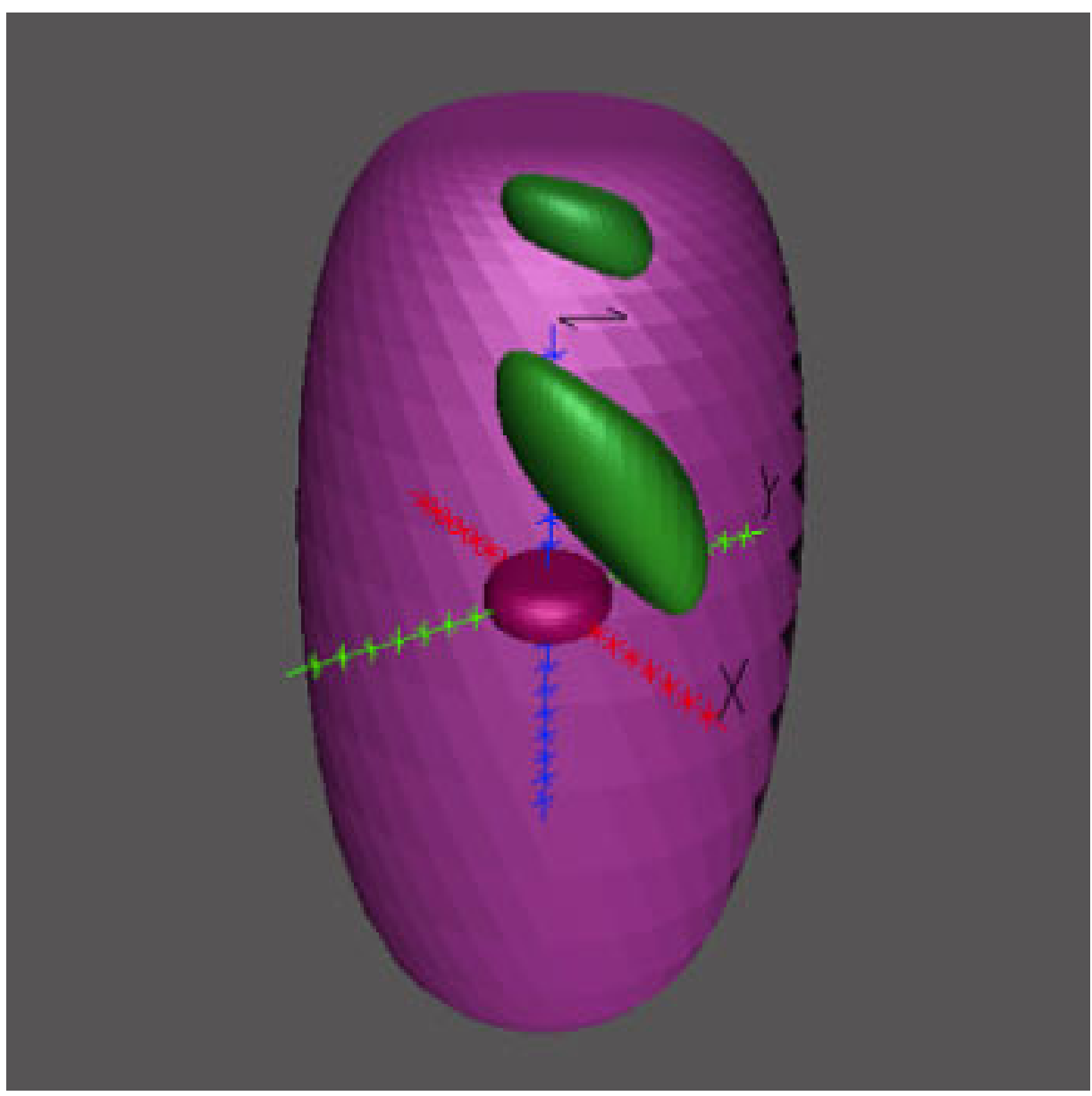

In this illustration, the first generator is again a dilation with fixed points at 0 and infinity in Heisenberg coordinates. However, this time its dilation factor is only 3.5. The fixed points of the second generator have also been perturbed off of the vertical chain. They lie at approximately $(.2,3)$ and $(.48-.16 I, 1.58)$ in Heisenberg coordinates. The dilation factor of the second generator is also 3.5 .

These generators exhibit a more generic configuration of fixed points, but the group they generate lies closer to the boundary of Schottky space. As you can see, the red and green spinal spheres nearly touch, i.e. the equidistant bisectors they bound are nearly asymptotic.

\section{REFERENCES}

[1] K. Astala and G. J. Martin Holomorphic motions, preprint 1994.

[2] L. Bers, On boundaries of Teichmüller spaces and on Kleinian groups: I, Ann. of Math. 91 (1970), pp. 570-600. MR 45:7044

[3] L. Bers, Spaces of Kleinian groups, Lect. Notes in Math., no. 155, Springer-Verlag, Berlin, 1970, pp. 9-34. MR 42:6216

[4] S. S. Chen and L. Greenberg, Hyperbolic spaces, in Contributions to Analysis (A collection of papers dedicated to Lipman Bers) (ed. L. V. Ahlfors et al.), Academic Press, New York, 1974, pp. 49-87. MR 51:13934 
[5] K. Corlette, Hausdorff dimension of limit sets, I, Invent. Math. 102 (1990), pp. 521-542. MR 91k:58067

[6] C. J. Earle, I. Kra and S. L. Krushkal', Holomorphic motions and Teichmüller spaces, Trans. Amer. Math. Soc. 343 (1994), pp. 927-948. MR 94h:32035

[7] W. M. Goldman, Complex hyperbolic Kleinian groups, in Proceedings of the Osaka International Conference, (eds. G. Komatsu and Y. Sakane), Lect. Notes in Pure and Appl. Math., no. 143, Marcel Dekker, Inc., New York, Basel and Hong Kong, 1993, pp. 31-52. MR 93k:32053

[8] W. M. Goldman, Introduction to Complex Hyperbolic Geometry, Oxford University Press, to appear.

[9] W. M. Goldman and J. Parker, Complex hyperbolic ideal triangle groups, J. Reine Angew. Math., 425 (1992), pp. 71-86. MR 93c:20076

[10] W. M. Goldman and J. Parker, Dirichlet polyhedra for dihedral groups acting on complex hyperbolic space, J. of Geom. Analysis, 2 (1992), pp. 517-554. MR 94d:32044

[11] V. Guillemin and S. Sternberg, Symplectic techniques in physics, Cambridge University Press, 1984. MR 86f:58054

[12] J. Heinonen and P. Koskela, Definitions of quasiconformality, Invent. Math. 120 (1995), pp. 61-79. MR 96e:30051

[13] T. Jørgensen, On discrete groups of Möbius transformations, Amer. J. Math. 98 (1976), pp. 739-749. MR 55:658

[14] A. Korányi and H. M. Reimann, Quasiconformal mappings on the Heisenberg group, Invent. Math. 80 (1985), pp. 309-338. MR 86m:32035

[15] A. Korányi and H. M. Reimann, Contact transformations as limits of symplectomorphisms, C. R. Acad. Sci. Paris 318, série I (1994), pp. 1119-1124. MR 95j:53049

[16] A. Korányi and H. M. Reimann, Foundations for the theory of quasiconformal mappings on the Heisenberg group, Adv. in Math. 111 (1995), pp. 1-87. MR 96c:30021

[17] G. J. Martin, On discrete isometry groups of negative curvature, Pacific J. of Math. 160 (1993), pp. 109-127. MR 94g:57041

[18] B. Maskit, Kleinian groups, Grundlehren der math. Wiss., no. 287, Springer-Verlag, 1988. MR 90a:30132

[19] J. Mitchell, On Carnot-Carathéodory metrics, J. Diff. Geom. 21 (1985), pp. 35-45. MR 87d:53086

[20] G. D. Mostow, Strong rigidity of locally symmetric spaces, Ann. of Math. Studies, vol. 78, Princeton Univ. Press, Princeton, N.J., 1973. MR 52:5874

[21] P. Pansu, Métriques de Carnot-Carathéodory et quasiisométries des espaces symétriques de rang un, Ann. of Math. 129 (1989), pp. 1-60. MR 90e:53058

[22] J. G. Ratcliffe, Foundations of hyperbolic manifolds, Graduate Texts in Math. 149, SpringerVerlag, 1994. MR 95j:57011

[23] P. Tukia, On isomorphisms of geometrically finite Möbius groups, Publ. Math. IHES 61 (1985), pp. 171-214. MR 87j:30110

[24] J. Väisälä, Lectures on n-dimensional quasiconformal mappings, Lecture Notes in Math., no. 229, Springer-Verlag, 1971. MR 56:12260

Leica AG, PPT 4199, 9435 Heerbrugg, Switzerland

E-mail address: Beat.Aebischer@email.leica.com

The Geometry Center, University of Minnesota, Minneapolis, Minnesota 55454

E-mail address: rminer@geom.umn.edu 